\title{
Is a routine preoperative rectosigmoidoscopy necessary in patients with bilateral inguinal hernia?
}

\author{
Akbaba S, Ersoy E, Gundogdu H, Yasar U \\ Ataturk Research and Educational Hospital, Department of General Surgery, Ankara, Turkey. \\ perenersoy@gmail.com
}

\begin{abstract}
Objectives: The purpose of this prospective study was to evaluate the association between bilateral inguinal hernias and colorectal cancers.

Purpose: Inguinal hernias are one of the most common subjects in surgical practice and have been known to be associated with some other pathologies since 1831. Although there are some series in literature reporting the association of colorectal cancers with inguinal hernias, it is still controversial to perform colorectal diagnostic tools in hernia patients. Colorectal cancers are particularly accused to be in association with synchronous bilateral hernias as they increase the intra-abdominal pressure.

Methods: Rectosigmoidoscopy was performed in 110 consecutive bilateral hernia patients and the results were recorded prospectively. Patients having colorectal diseases were excluded.

Results: There were no pathologies in $87(\% 79,1)$ rectosigmoidoscopies, while benign pathologies (hemorrhoids, polyps and diverticulitis) were diagnosed in 23 (\%20,9).

Conclusions: It has not been proved yet that colorectal cancers increase the incidence of bilateral inguinal hernias. The incidence of benign pathologies in our series was similar to that of same age population without hernia. As a conclusion of this study we believe that rectosigmoidoscopy is not necessary for synchronous bilateral hernias unless the patient has any complaints or risk factors. Colorectal screening tools are performed when the clinical findings or the story of the patient support colorectal cancers) (Tab. 1, Ref. 25). Full Text in PDF www.elis.sk. Key words: bilateral inguinal hernias, rectosigmoidoscopy.
\end{abstract}

In 1831, Sir Astley Cooper noticed the association between inguinal hernia, one of the very common diseases subjected to surgical procedure today, and certain chronic gastrointestinal disorders (1). In 1963 Terezis et al reported in their series of 107 colon cancer that $22.5 \%$ of them had inguinal hernia at the time of diagnosis or within 2 years (2). After that, some researchers began to recommend routine preoperative colorectal screening in patients with inguinal hernia (3-7). Then this association was questioned in some studies, and data had not verified the results $(8-11)$. However, there have been no studies published on the association between sudden bilateral inguinal hernias and colorectal pathologies. A prospective study was planned to investigate this issue.

\section{Materials and methods}

Ethical committee approval has been obtained for the study to assess the patients with sudden inguinal hernia by rectosigmoidoscopy. Between 2005-2010, 110 consecutive patients presented with bilateral inguinal hernia and in preparation for operation underwent preoperative rectosigmoidoscopy after obtaining an informed

Ataturk Research and Educational Hospital, Department of General Surgery, Ankara, Turkey

Address for correspondence: Eren Ersoy, MD, Acar Beytepe Evleri, 06800, 154/27, Beytepe, Ankara, Turkey.

Phone: +90.532 .3868600$ consent. Patients were excluded if they had any gastrointestinal complaint, medical history of colorectal disease and family history of polyp or cancer. The clinical and colorectal screening findings were recorded, and the results were followed prospectively.

\section{Results}

The study population consisted of 108 male (98.18\%) and 2 female $(1.82 \%)$ patients with a median age of 50.24 years (range 25-79 years, median: 52$)$. Whereas 87 patients $(79.1 \%$ ) had normal rectosigmoidoscopy findings, 23 had $(20.9 \%)$ various benign pathologies, including hemorrhoids in $13(11.8 \%)$, hyperplastic polyps in $6(5.5 \%)$, adenomatous polyps in $4(3.6 \%)$, and diverticulosis in $4(3.65 \%)$ patients. None of the patients had malignancy. All these pathologies except hemorrhoid were found in patients over 40 years of age.

The most common comorbidities were prostate diseases (20\%), chronic pulmonary diseases $(12.7 \%)$, and hypertension $(12.7 \%)$. While 96 of the patients had primary inguinal hernia $(87.3 \%), 10$ had $(9.1 \%)$ primary on one side and recurrent on the other, and 4 patients had (3.6\%) bilateral recurrent inguinal hernia. In addition, preoperatively undetected femoral hernia was found in 2 patients during operation (1.8\%). Sixty patients $(54.5 \%)$ received general anaesthesia and $50(45.5 \%)$ epidural anaesthesia, and all of them underwent bilateral Lichtenstein hernia repair. None of the patients developed a postoperative major problem. 
Tab. 1. The results of colorectal screening studies in patients with inguinal hernia.

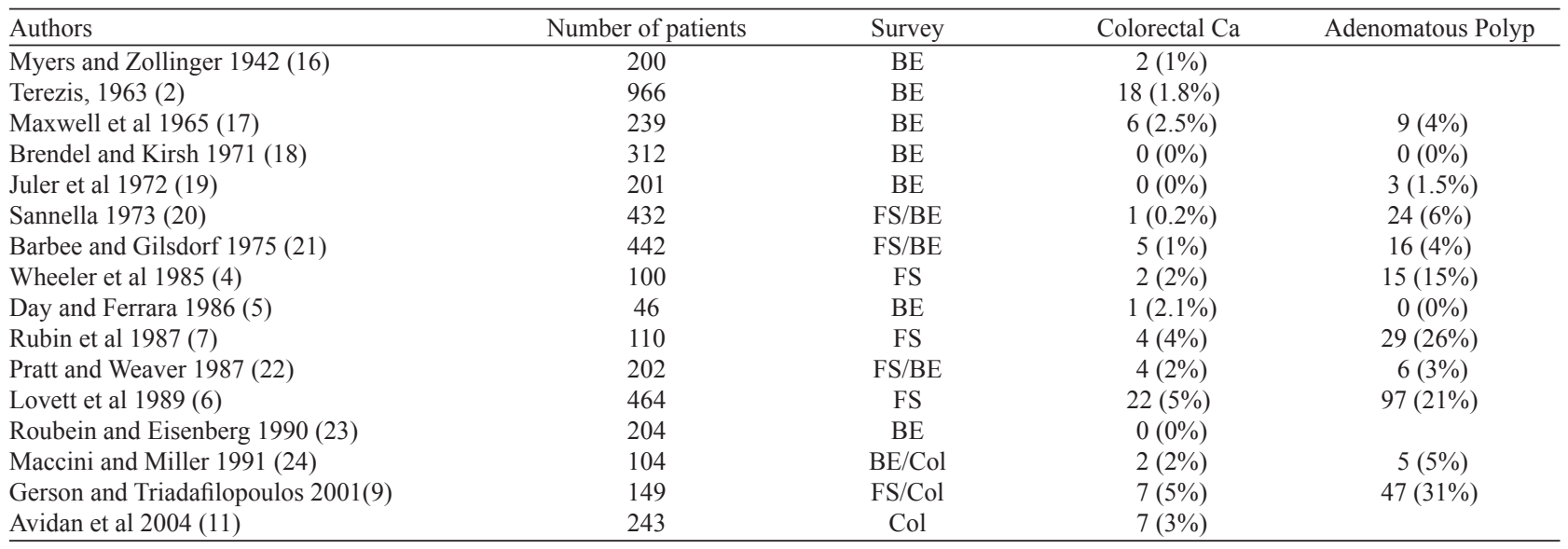

BE - Barium Enema, FS - Flexible Sigmoidoscopy, Col - Colonoscopy

\section{Discussion}

Inguinal hernia and colorectal cancer are more frequent in men and elderly population $(4,10,12,13)$. Inguinal hernias are more common in men, thought to be related to the anatomical differences between the male and female pelvis. In general, the male pelvis is narrow, the pubic arch is lower, the inguinal canal is shorter, and the shutter mechanism is considerably weaker (14). With increased age, in addition to weakening of support tissue, obstructive uropathy, severe coughing, and chronic pulmonary diseases facilitate the development of inguinal hernia (10). It is also reported that smoking, long-term heavy labor work, the diseases characterized by abnormal collagen production, and abdominal aorta aneurysm play a role in inguinal hernia development (14).

The publication of studies indicating the association between colon cancer and inguinal hernia has led to the idea of pre-operative colorectal screening (2-7). Studies indicated that inguinal hernia occurred in approximately $17-22 \%$ of male patients with colorectal cancer, or inguinal hernia was detected within 1-2 years after cancer diagnosis and, therefore, underlined a need for colorectal screening as part of preoperative evaluation. This association was considered to be related to partial luminal obstruction of colorectal pathologies, and increased intra-abdominal pressure due to impaired motility and constipation, which was forcing the weak points of abdominal wall. In addition, increasing abdominal discomfort made patients more sensitive to the changes in the abdominal wall and motivated them for a repair (4). The views trying to explain the pathophysiology and the lack of studies about sudden bilateral inguinal hernia in literature led us to study this issue.

On the other hand, approximately $25 \%$ of individuals around 50 years of age have adenomatous polyposis and $5 \%$ have colorectal cancer in the general population, and the prevalence is known to increase with age $(13,15)$. It is believed that the lifetime risk of colorectal cancer is approximately $5 \%$. Table 1 shows the colorectal screening results of the patients with inguinal hernia published by various researchers. As this table suggests, the incidence of colorectal cancer is between $0-5 \%$, and that of adenomatous polyps is $0-31 \%$. These rates are not above the values that are detected in the screening of 50 years of age individuals in normal population. Therefore, it has not been proven that the patients with inguinal hernia have shown a higher rate of colorectal neoplasm or precancerous lesions compared to normal population.

In this study, we determined that $20.9 \%$ of the patients had pathological findings, and when hemorrhoids were excluded, the rate of the other findings was $9 \%$. When we consider all these lesions are seen in patients over age 40 , we may say that our rates are similar to the incidences observed in normal population.

Although colorectal pathologies were not considered as a risk factor listed in European Hernia Society guideline, published in 2009, "the actual risk factors that could cause the development of hernia" were included among the suggested topics for future research (25). Therefore, this issue will remain on the agenda for a while.

It is very important to screen the population at risk for colorectal cancer, which is the second most common cause of cancer deaths after lung cancer. Screening of asymptomatic persons with an average risk of cancer will decrease the mortality rate by providing early detection of cancers in curable period, as well as the incidence of cancer by eliminating precancerous lesions.

We have concluded that in patients with bilateral inguinal hernia, even if of a sudden, routine colorectal screening is not necessary if they do not have symptoms or risk factors. Colon screening should be done only if supported by clinical findings and medical history and a full colonoscopy should be preferred.

\section{References}

1. Juler GL, Stemmer EA. Inguinal hernia and colorectal cancer. Hernia. Nyhus LM, Condon RE (Eds). Philadelphia: JB Lippincott Co, 2nd Ed, 1978, pp 95-96.

2. Terezis NL, Davis WC, JacksonFC. Carcinoma of the colon associated with inguinal hernia. N Eng J Med 1963; 268: 774-776.

3. Davis WC, Jackson FC. Inguinal hernia and colon carsinoma. CA Cancer J Clin 1968; 18: 143-145. 
$604-606$

4. Wheeler WE, Scott-Conner CEH, Stone RA. Flexible sigmoidoscopy as a screening procedure for asymptomatic colorectal carsinoma in patients with inguinal hernia. Southern Med J 1985; 78 (12): 1417-1420.

5. Day T, Ferrara JJ. Preoperative barium contrast enema in patients with inguinal hernia. Southern Med J 1986; 79 (11): 1339-1341.

6. Lovett J, Kirgan D, McGregor B. Inguinal herniation justifies sigmoidoscopy. Am J Surg 1989; 158: 615-617.

7. Rubin BG, Ballantyne GH, Zdon MJ et al. The role of flexible sigmoidoscopy in the preoperative screening of patients with inguinal hernia. Arch Surg 1987; 122: 296-299.

8. Birolini C, Utiyama E.M, Rodrigues AJ, Birolini D. The associated of inguinal hernias and colon cancer. A technical strategy based upon three patients. Hernia 1998; 2: 139-143.

9. Gerson LB,Triadafilopoulus G. Is colorectal canser screening necessary in the preoperative assessment of inguinal herniorrhaphy? A casecontrol study. Am J Gastroenterol 2001; 96 (6): 1914-1917.

10. Avidan B, Sonnenberg A, Bhatia $\mathbf{H}$ et al. Inguinal hernia is not a sign of colon cancer: results of a prospective screening trial. Aliment Pharmacol Ther 2002; 16: 1197-1201.

11. Avidan B, Bardan E, Lang A et al. Colorectal cancer screening in patients presenting with an inguinal hernia: is it necessary? Gastrointestinal Endoscopy 2004; 59 (3): 369-373.

12. Grenlee TR, Murray T, Bolden S, Wingo PA. Cancer statistics 2000. CA Cancer J Clin 2000; 50: 7-33.

13. Lieberman DA. Screening for colorectal cancer. N Eng J Med 2009; 361 (12): 1179-1187.

14. Van Wessem KJP, Simons MP, Plaisier PW, Lange JF. The etiology of indirect inguinal hernias: congenital and/or acquired? Hernia 2003; 7: 76-79.
15. Winawer SJ, Fletcher RH, Miller l et al. Colorectal cancer screening: clinical guidelines and rationale. Gastroenterology 1997; 112: 595-642.

16. Myers RS, Zollinger RM. Gastrointestinal symptoms and inguinal hernia. N Eng J Med 1942; 227: 660-661.

17. Maxwell JW, Davis WC, Jackson FC. Colon carcinoma and inguinal hernia. Surg Clin North Am 1965; 45: 1165-1171.

18. Brendel TH, Kirsh IE. Lack of association between inguinal hernia and carcinoma of the colon. N Eng J Med 1971; 284: 369-370.

19. Juler GL, Stemmer EA, Fullerman RW. İnguinal hernia and colorectal carcinoma. Arch Surg 1972; 104: 778-780.

20. Sannella NA. Inguinal hernia and colon carcinoma: presentation of a series and analysis. Surgery 1973; 73: 434-437.

Barbee CL, Gilsdorf RB. Results of routine preoperative sigmoidoscopy and barium enema on patients with inguinal hernia. Am Surg 1975; 41: 28-31.

21. Pratt SM, Weaver FA. Preoperative evolution of patients with inguinal hernia for colorectal disease. Surg Gynecol Obstet 1987; 165: 53-56.

22. Roubein DR, Eisenberg RL. Inguinal hernia and colon cancer. South Med J 1990; 83 (7): 855.

23. Maccini DM, Miller RM. The yield of barium enema in patients undergoing inguinal hernia repair or abdominal hysterectomy. Surg Gynecol Obstet 1991; 172: 391-393

24. Simons MP, Aufenacker T, Bay-Nielsen M et al. European hernia society guidelines on the treatment of inguinal hernia in adult patients. Hernia 2009; 13: 343-403.
Received May 13, 2011. Accepted June 26, 2012. 